\title{
Il. JAIIRESBEIRICITE.
}

\section{Römische historiker der kaiserzeit.}

(S. Philol. XXXII, p. 155. 541.)

\author{
Zweiter artikel ${ }^{1}$ ). \\ J u l i us Flor us.
}

1. Iuli Flori epitomae de 'Tito Livio bellorum omnium annorum DCC libri II. Recensuit et emendavit 0 t t o J a hn. Lipsiae apud Weidmannos. MDCCCLII. XLVIIII et 136 pp. 8. (Rec. von K. Halm Jbb. f. Plilol. 1854, LXIX, 172-196).

2. Iuli Flori epitomae de Tito Livio bellorum omnium anuorum DCC libri duo. Recognovit Carolus Halm. Lipsiae sumptibus et typis B. G. Teubneri. MDCCCLIII. $X X$ et 125 pp. 8.

3. F. Bölımer, Zur kritik des Florus: Jalıns Archiv f. philol. 1853, XIX, 636-638.

4. Th. ll o m msen, Kritische miscellen: Berichte der k. säclıs. ges. d. w. philol. bist. kl. 1854, VI, p. $(153-160) 156 \mathrm{f}$. $659 \mathrm{f}$.

5. J. Ilälly, zu Florus: Jbb. f. philol. 1857, LXXV,

6. H. G. Plass, De auctoribus eius quae vulgo fertur $I$. Annaei Flori epitome rerum romanarum: Progr. des Domgymnasiums zu Verden. 1858, 8. p. 7-16.

7. U. Kölı ler, Qua ratione T. Livii annalibus usi sint listorici latini atque graeci describitur et quid inde in Livii textu quem dicunt constituendo repeti possit exponitur et exemplis illustratur. Commentatio philologica . . NDCCCLA praemio regio ornata. Gottingae III et $99 \mathrm{pp}$. 4 .

8. L. S p e n g e l, Ueber die geschichtsbücher des Florus, vorgetr. in der sitzung der philos.-philolog. classe 7. Juli 1860. Aus den abhandl. J. k. bayer. ak. J. w. I. cl. IX. bd. II. alstlı. Nlünclien 1861 (G. Franz) 34 s. 4.

1) Recensionen siud nur ausnahmsweise augeführt, wenn durch vie die forschung gefördert worden ist. Schriften, welche nur nebenher von Florus handeln, sind ausgeschlossen oder höchstens gelegentlich erwähnt. 
9. Th. Mo m m se n, Handschriftliches zu Florus : Rhein. mus. f. philol. N. F. XVI, p. 135 .

10. A. Kellerhauer, $Z u$ den römischen historikern. Ill. Julius Florus: Philologus XXI, p. $160 \mathrm{flg}$.

11. F. E. K ölıle r, Observationes criticae in Inlium Florum. Dissertatio inauguralis ... Gottingae MDCCCLXV. $42 \mathrm{pp} .8$.

12. J. Relier, Das geschichtswerk des Florus. Progr. der k. [bayer.] studienanstalt Freising. 1865. VI und 71 s. 8.

13. C. He y n, De Floro listorico. Dissertatio philologica ... MDCCCLXVI. Bonnae. 53 pp. 8.

14. L. Vielh aber, Zu Florus, Valerins Maximus und Caesar: Zeitschr. für die österreich. gymn. XVIII, 244-253.

15. J. Freudenberg, Kritische bemerkungen zu Florus und den periochae des Livius: Rhein. mus. f. philol. N. F. XXII, p. $25-30$.

16. J. P. B insfeld, Zu Florus: Rhein. mus. f. philol. N. F. XXII, p. 340 und ebend. 645 .

17. J, P. Binsf eld, Quaestiones Florianae criticae. Progr. d. gymn. zu Disseldorf. $1869.9 \mathrm{pp.} 4$.

18. E. Ballens, Lectiones latinae. Dissertatio philologica ... NIDCCCLXX. Bonnae. 8. p. 5-19). (Rec. von H. S.: Philol. Anz. III, 125-129.

19. E. Wölffli n, Stilistische nachahmer des Tacitus: Philologus XXIX, p. $557 \mathrm{f}$.

20. F. v. 1l out, Zu Florus: Jbb. f. philol. 1870, Cl, p. 79 f.

21. H. Müller, Zur kritik des Florus: Rhein. inus. $f$. philol. N. F. XXVI, p. 350 if.

22. H. Sa up e, Commentatio de arte critica in Flori bellis recte facienda. Ind. schol. . . Gottingae [MDCCCLXX] 4. p. $3-21$.

23. H. Müller, Zur kritik des Florus: Jbb.f. philol. 1871, Cll, p. 565-575.

Der bericht über die theilweise höchst bedeuteudeu leistungen, durch welche seit zwei jahrzehnten die kritik und exegese des Florus in einer weise gefördert worden ist, wie es nur selten bei einem schriftsteller niederen ranges geschieht, hebt naturgemäss mit der linweisung auf diejenige ausgabe an, welche nach dem ausspruche des competentesten beurtheilers (Halm nr. 1, p. 195) im vollen sinne des wortes den namen einer clitio princejs verdient. Seit dem ersten erscheinen der sogenannten epitome des Florus (Paris 1470) war der text auf solche landscliriften gegründet, welche von Otto Jahı (nr. 1) mit bestimmtleit als interpolirt bezeichnet werden konnten; weder Jo. Camers (Wien 1518) noch El. Vinetus (Poitiers 1554) noch Jan. Gruter (Heidelberg 1597) haben daran etwas wesentliches geändert. Und obschon der letztere sowie Cl. Silmasius (Heidelberg 1609) den werthvollen 
codex Nizairianus $(N)$ benutzen konnten, so war dadurch noch nicht eine bessere grundlage des textes, sondern nur der rehitiv beste vertreter der bereits geltenden grundlinge gefunden. Der werth der wiederholt infgelegten sammelausgaben von J. G. Graevius (Utrecht 1680) und C. A. Duker (Leyden 1722) berulit aber melır aut der erklärung. Endlich hait zwar G. Seebode (Leipzig 1821) die Bamberger handschrift $(B)$, welche eine wesentlich verschiedene überlieferung repräsentirt, benutzt, ohne sie jedoch weiter als in einzellheiten $z$. b. der wortstellung bei seiner recension zur richtschnur zu nelomen. Erst 0 t t o J a h n (nr. 1) war es vorbehalten, den ganzen gewinn, welcher sich aus dieser handschrift für die herstellung des Florustextes ergibt, zu schöpfen, nachdem schon K. Lachmann und $K$. Halm die bedeutung der handsclırift unablängig von einander erkannt hatten (nr. 1, p. 1II). Es würde wenig bedeuten, wenn von Jalıns ausgalbe gerühmt würde, dass sie einen wirklichen fortschritt bezeichne. Deun es gehörte in der that nicht viel dazu die ausgabe von Seiht (Prag 1847) zu übertrelien, deren verfasser seine literaturkenntniss schon in der vorrede genügend charakterisirt, indem er von einem Privilegium (sic!) Veneris spricht. Aber Jahns leistung ist als mustergültig für die kritik überlianpt vielfaltig und olne widerspruch anerkinnt worden. „Denn jedes bei erzielung eines wirklichen durch eine ausgabe zu erwirkenden fortschrittes nothwendige gescläft sagt E. v. Leutsch im Philol. Anz. Il, p. 12 - fülırt der verfasser hier mit grösster umsiclit und sorgfalt ius: die himdschriften sind genau verglichen und ilıre verliältnisse bis ins einzelnste dargelegt, die sprache des schriftstellers und die seiner zeit wie der von ihm behandelte stoff nach allen richtungen durchforscht, daher einerseits die gebührende achtung vor der überlieferung, indrerseits scharfsinniges erkennen des verdorbenen und mit hülfe überraschender combination glücklichste heilung durch conjectur - in der einleitung speciellste kenntniss des lebens des scliriftstellers und seines werks und die da mülssim gefundenen resultate anspruclislos und doch elegant dargestellt, überall das schönste den classikern alogelauschte matasshalten“" Wenn im folgenden demnoch nicht nach der Jalın'schen, sondern nach Halm's amsgabe citirt wird, so ist das durch die mannichfaltigen nachbesserungeu gerechtfertigt, die $\mathrm{H}_{i 3} \mathrm{~m}$ in seiner recognition des textes geboten hiat, wie denn schon Jalun's text seine durchiarbeitung nicht zum geringsten theile ,durch den antheil, welchen Mommsen, Hasut, Halm daran nalmen, erreicht"“ hatte (vgl. Vahlen, Otto Jalın P.10). Folgendes sind diejenigen punkte, wodurch Halm's text sich namentlich von dem Jaln'schen unterscheidet: die angabe einiger guten von Seebode zuerst aus $B$ aufgenommenen lesarten, welche bei Jahn übersehen worden waren, ist von Halm nachgetragen. Die mittheilung der schon bei Jahn verzeichneten lesarten in $B$ ist auf grund wiederholter ein- 
sicht der haudschrift in einzelnem, insbesondere bezüglich der rasuren sowie der unterscheidung einer ersten und zweiten hand berichtigt. Vereinzelte stellen sind aus einer von Jahn nicht benutzten collation eines codex Emmeranus des Jordanes verbessert, andere, in denen Jahn der autorität von $B$ gefolgt war, auf grund des zeugnisses der Jordaneshandschriften allein oder dieser im verein mit der zweitbesten Florushandschrift $N$ liergestellt worden. Wieder andere stellen hat Halm durch eigene conjectur oder nach den ihm mitgetheilten vermuthungen seiner freunde Bezzenberger, Mommsen, L. Spengel, Wölfflin und des frühverstorbenen Eduard Wurm berichtigt. Aber ,der hauptsächlichste differenzpunkt - wie Halm selbst (nr. 1, p. $195 \mathrm{f}$.) angibt - bestelit darin, dass ref. [Halm] nach nochmaligem studium der Bamberger haudschrift zu der überzeugung gelangt ist, dass der text des Florus sich noch etwas genauer nach diesem codex herstellen lasse, als es von Jahn bereits geschehen ist". In diesem punkte ist inzwischen von verschiedenen seiten widerspruch erhoben worden, am eindringlichsten von H. Sauppe (nr. 22), welchem H. Müller (nr. 23) beigestimmt hat, nachdem schon vorher durch Sauppe aufmerksim gemacht F. E. Kohler betont hatte, dass $B$ obwohl vorzüglicher als $N$ doch auch selbst so vielfach interpolirt sei, dass er nur eine ungenügende grundlage für die kritik des textes biete. Jahn hatte in der vorrede seiner ausgabe p. XII folgende methode der textesrecension festgestellt: Arlis crilicae paullo peritiores non dubitabunl, credo, quin codicibus recentioribus omissis $B$, alhibilo ubi fieri potest I [i. e. lordane], fundamentum verae lection is restituendue habendus sit. sed vel optimus codex non inmunis a vitiis est quae emendatione egent, sive ex antiquioribus libris translata sint, sive ex librarii incuria nala . ad quae deprehendenda atque tollenda permagni momenti est codex qualem $N$ habemus, qui quamquam in aniversum inferior est, hand panca tamen sive casu sive librarii diligentia recte scripta servavil quae in $B$ corrupta extant. Dieselbe methode wurde von Halm nur noch consequenter durchgeführt, auch von I. Spengel (ur. 8) thatsachlich durchaus gebilligt und $B$ (z. b. p. 27 anm.) ausdrücklich als "beste haudschrift" gegenüber dem codex $N$, der "mitunter arge interpolationen erfahren hat", bezeichnet. Nach Siuppe (nr. 22, p. 17) würde jedoch die werthschaitzung der beiden handschriften und demnach iluch das kritische verfaliren in folgender weise zu modiliciren sein. Ostendisse nobis, quod volebamus, videmar, fere parem asse et in nazariano et in bambergensi codice et bonarum lectionum et locorum vel negligentia alque errore vel interpolationibus corruplorum, ita ut de verilate lectionis alicuius indicaturi non anctoritate codicis alterius utrius confisi, sed rationibus ubique rerum ipsarum, hoc est vel sententiarum vel sermonis, ducti decidere debeamus. Saupe selbst hat es (nr. 18, p. 128) als seine iberzeugung ausgesprochen, 
dass durch seine beweisfulirung die ansiclıt "beseitigt" sei, dass man sich im Florus nur necessitate qmadam cogente, wie Bährens (ur. 18, p. 14) sagt, von $B$ entfernen dürfe. Lis wird die aufgrabe des die emendation des Florus betreflenden theiles dieser berichterstattung sein, durch besprechung einer inswalıl bezeichnender stellen für diese frage die entscheidenden gesiclitspunkte darzulegen. Einstweilen bedurfte es dieser orientirenden vorbemerkung um zu beweisen, dass jedenfalls für die untersuchung über den namen des schriftstellers und, was daunit zusimmenlängt, äber die persönlichkeit und das zeitalter desselben, neben dem zengnisse in $B$ anch das von $N$ in betraclit zu zielten ist.

Der name des schriftstellers ist in $B$ als Julius Florus, in $N$ und den mit diesem codex verwandten jungeren liandschriften als $I$. A n in a e i s Flo r u s überliefert. Die entscheidung, welche form des namens die echte sei, wird durch das zusiummentreffen melırerer spuren von ilınlichen nimen, deren träger mit dem verfasser der epitome abwechselnd identificirt wurden, nicht erleichtert, sondern erschwert. Schon lange bevor aus $B$ der name Julius Florus bekannt geworden war, hatte Iipsius, der sich jedoch fïr den namen Annuens entschied, nach einer vermuthung des Riphinel Maffeius Volaterranus und anch der ältere Vossius an Julius Florus, einen solın des von Quintil. X, 3, 13 erwälnten Iulius Florus, in elogrentia Galliarum princeps, gedacht, welcher letztere vielleicht mit dem bei Seneca Controv. VIIII, p. 258 Bu. (p.409 Kiessl.) genannten identisoh ist. F. N. Titze in seinen wunderlichen untersuchungen de epitomes.. aetale probabilissima, vero anctore, operis antigua forma (Linz 1804 ) hatte unter der voraussetzung einer beispicllusen interpolation den angeblich allein echten bestand des textes anf Iulius Florus, den bekinnten zeilgenossen und freund des dichters Horitius, zuriickgefuhrt und dieselbe ansicht in seiner ansgabe (Prang 1819) wiederholt. Doch weder diese abentenerliche behauptung, welche von Meinert (Wiener Jihrbb. XXVIII, 169-201) und besonders von Gossrau (Quedlinburger progr. 1837 de Flori qua vixerit aetate) eine gründliche zurückweisung erfalıren hit, noch jene erstere vermuthung verdienen irgend welche beaclitung. Vebrigens hat Plass (nr. (i) Titze's ansicht theilweise wieder erneuert, indem auch er denselben Julius Florus als den verfasser der epitome betrachtet, da er sich uur so die lobeserhebungen des Augustus, den inmitten dieser regierungszeit alogebrochenen schluss des werkes, gewisse eigenthümliclıkeiten der composition desselben, endlich einzelne aufliallende stellen erklären kann. Allein keiner dieser gründe ist stichlaaltig : daus lob des Augustus erklärt sich aus dem abschen des Florus gegen die bürgerkriege; Jer schluss des werkes aber spricht eleer gegen als für die annabme von Plass; denn ein zeitgenössischer lobredner des Augustus würde seine erzälılung des lecllum Germanicum II, 30 wohl kaum mit der niederlage des Varus 
und der betrachtung geschlossen haben: hac clade factum est, ut imperium, auod in litore Oceani non steteral, in ripa Rheni fluminis staret. Ueber die composition der epitome wird weiter unten die rede sein. Jene allerdings auffallenden stellen aber, besonders II, 30, 38, haben schon bei Jahn (ur. 1 p. XLVI), dessen ausgabe freilich Plass ebenso wenig als die Halm'sche zu kennen scheint, genügende aufklärung gefunden. Statt aber wie 'Titze die mit sicherheit auf eine spaitere abfassungszeit hinweisenden stellen als iuterpolirt auszuscheiden, greift Pliass (p. 15) zu folgender Panacee: postero tempore rhetor aliquis vanus, fortasse adolescentulus ludens illa laudatione usus est, quam in rerum romanarum epitomen per quatuor vilae aetates descriptam permutaret, nec tamen incepta absolvit. Widerlegung erscheint hier unnöthig. Nur das zeugniss des codex $B$ spricht wirklich für den namen Julius. Auch sagt G. J. Vossius de list. lat. lib. I, cap. 30 : Florus, uti in plerisque libris Inlius, ita Annaeus in vell. vocatur libris und ebenda: ut in nonmullis libris legere est, L. Iulius Florus. Unter den äbrigen handschriften, welche den namen $L$. Amacus bieten, gibt eine Heidelberger 1568 (350) folgende, wie schon Salmasius salı, auf Hieronymus zurückzufülirende notiz: Anno XIII olympiadis (vielmelır III olympiadis CCXI) Lucius Anneus Melas fr. Senecae et Gallionis bona Lucani poctue filii sui a Nerone promeruit - und knüpft daran die combination, dass entweder Melas oder Seneca den namen Florus gehabt habe und verfasser der cpitome sei. Auf diese trübe quelle fülırt Jahn anch mit recht die in einigen jüngeren landschriften der überschrift beigefügte bezeichnung Seneca zurück. Uebrigens sclieint auf diesen namen noch ein anderes zeugniss himzuleiten. Es heisst nemlich bei Lactantius Institutt. div. Vil, 15, 14 non inscile Seneca Romanac urbis tempora distribuit in actates; und da dieselbe parallele zwischen den lebensiltern des römischen volkes und eines einzelnen menschen auch im prologe des Florus wiederkehrt, so war es nicht allzukïhın, trotz einzelner zuerst von Vossius a. o. hervorgehobenen verschiedenleiten in der durchfübrung jener analogie beide versionen des gleichen grundgedankens zu ideutificiren und so aus der stelle des Lactantius die autorschaft des Seneca für die epilome abzuleiten. Wälırend nun Jahın wie seit Vossius die meisten die identitït der von Lactantius verwertheten stelle und der entsprechenden partie der epitome bestreitet und vielmelır vermuthet, diss Florus den bei Seneca vorgefundenen vergleich nur rhetorisch ausgemalt habe: verstelit er unter Seneca nicht den philosophen, sondern dessen vater, der nach dem zenguisse des solsnes (de vita patris fr. $X V$, 98) historiac ab initio bellorum civilium geschrieben hatte. Is igilur cum senectutem Romae, quam a bellis civilibus incipientem fecil, descripsisset, Florus infantiam adulescentiam iuventutem sibi sumpsit. So Jalun. Dagegen findet Spengel (nr. 8, p. 2! f.), 
dass ,die nücliternheit dieses mannes, den wir [aus den suasorien und controversien] linreichend kennen, einer solchen auffassung entgegen" stehe. Aler auch dem philosophen Seneca sei diese originelle denkweise und metaphorische sprache ebenso fremd, wie sie dem Florus angeboren sei. An diesen erinnere schon die wiederholung des auch in der epitome allzu haufigen quasi. „Der gedinke selbst - sagt Spengel — dis ganze leben und weben des gesiummten römischen volkes mit dem einzeluleben des menschen auf gleiche linie zu stellen und nach diesem zu messen ist ein bild, unsers antors [Florus] vollkommen würdig, da sein ganzes werk von solchen vergleichungen strotzt und gerade darin die eigenthiumlichkeit seines buches bestelit". Iatantius will nach Spengels richtiger darlegung aus jener entlehnten vergleiclung nur das ergebniss gewinnen, dass auf die seneclus des römisclien volkes niturgemäss der tod, wie ilın anch die propheten verkündet, folgen müsse. „Desswegen ist alles von ilum erwähnt; die innere vertheilung von der infantia bis zur seneclus, worin die [hauptsächlichste] abweichung [zwischen dem citat des Lactantius und dem wortlante bei Florıs] bestelıt, ist nebensache und der jetzt in lö̈herem sclıwärmende kirchenvater konnte dieses, wie es sich seinem gedächtnisse aus der crimnerung darbot, ausführen, so dass einzelne abweichungen noch niclit den beweis einer fremden quelle geben. Hatte aber sein codex die aufschrift wie $N$ L. Annaeus (vielleicht selbst ohne Florus), so lag es nalie, da jener name der spatern zeit so bekannt wie der des M. Tullius lautete, den Seneca als den verfasser des buches anzufülıren". Dahler glaubt Spengel, dass Salmasius, welcher der überzengung ist, Lactantius habe nur Florus und keinen andern antor in ange gehaibt, unbefangener und richtiger geurtheilt habe, als Vossius und alle folgenden. Allein mag man auch dieser so wahrscheinlichen auffassung nicht beipflichten, sondern Jalun's erörterung für riclitiger halten: so viel ist klar, dass die für die autorsclaft des Seneca gezogenen consequenzen haltlos sind.

Ein Florus poeta, welcher mit dem kaiser Hadrian sclıerzhafte verse wecliselte, wird von Spartianus Hadr. Vit. 16 erwähnt, und es ist höchst wahrscheinlich, dass derselbe kein anderer ist als jener Aunius Florus, von welchem es bei Charisius I, p. 53, 14 (vergl. 140, 6) Keil. heisst: Annius Florus ad divum Hadrianum: poematis delector - und I, p. 123, 17 Florus ad divum Hadrianum: quasi de Arabe aut Sarmata manubias. Da nun früher bei Charisius Anneus gelesen wurde, so schien die annahme begründet, dass dieser dichter, auf den vielleicht nach dem codex Salmasianus auch einige kleinigkeiteu der Anthologie zurückzuführen sind, und der verfisser der epitome dieselbe person sei.

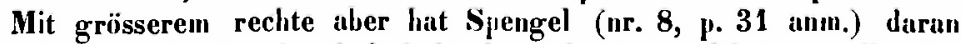
erinnert, wie charakteristisch in der zuletzt angeführten stelle das 
lieblingswort des Florus quasi erscheint. Ueber ein rhetorisches, nber in poetischem stile gehaltenes fragment $P$. Aunii Flori Virgilius orator an poeta wird in ersten inliange dieses berichtes referirt werden; einstweilen ist hier schon zu bemerken, diss Ritschl, welcher jenes bruchstïck zuerst herausgegeben hat, unter Jilhns beifall ausspricht, es sei kein grund vorhimlen zu bestreiten, dass der autor dieses tractates mit dem von Spartianus genannten F/orus pock und dem hei Clıarisius erwälınten Annius Florus identisch sei. Spengel (a. o.) erkennt in dem fragment die schöne, geschmuickte sprache und die haufigen ausrufungen des bistorikers wieder, findet aber auffallig, dass quasi und quippe nur je einmal in dem fragment vorkommen, was für diesen umfang viel zu wenig sei, da der listoriker jenes nicht weniger als 125 mal, dieses 75 mal gebrauclit.

Wie verlält sich nun dieser P. Annius Florus zu dem historiker I. Annaeus Florus, wie er in $\Lambda$ lieisst? Jahn sagt (praef. $\mathrm{XIN}$ ): nemo enim en usque temeritatis progredielur, ut aut hunc P. Annium aut illum L. Annacum vocandum esse contra omnes libros autumet; proesertim cum ille ipse L. Annaens Florus iam [codicis B anctoritate] incertus factus sit. Andere sind vor dieser ingeblichen tollkühnhleit nicht zurückgeschreckt. Halm (nr. 1, p. 192) theilt hierüber folgendes mit: ,anderer ansicht [als Jalın] ist .. Mommsen, der, wie er dem ref, mitgetheilt hat, nicht zweifelt, dass von dem verfasser des fragments auch die epitome herriilire, indem er annimmt, dass IVII FI,ORI in $B$ eine corruptel nus PVBIJI FLORI sei und andrerseits L. ANNEI, wie die geringern handschıriften des bistorikers lesen, aus P. ANNII. So kühn auch diese zurechtlegung der überlieferung erscheiıen mag, so sprechen für dieselbe doch ziemlich starke gründe: 1) die älnlichkeit der namen Anneus (Annacus) und Annius bei gleichem cognomen, 2) das genaue zusimmentreffen des zeitalters [siebe unten], 3) die schon von Ritschl hervorgehobene ühereinstimmung des stiles des fragments mit dem der epitome, wozu noch die ahuliclıkeit einzelner phrasen kommt, so besonders die lieblingswendung des epitomators per diversa terrarum, III, 6, 1. III, 19), 2 (vgl. anch in diversa terrarum II, 6, 41; per diversa gentium III, 5, 27), die auch in dem fragment $p$. XXXXII, 11 et. J. wiederkehrt; vgl. auch ill, victor gentinm populus in Fragm. p. XXXXI, 25 mit der Epit. III, 9, 3 victor gentium populus; III, 13, 2 populus gentium victor orbisque possessor; IV, 12, 61 victor gentium populus Romanus". Ausser Mlommsen und Halm hat auch Spengel, obschon ihm, wie es scheint, diese combination nicht entspricht, sich in der hanuptsache älnnlich entschieden; er sagt (a. 0. p. 30): "Woher die verschiedene benennung stammt, weiss ich so wenig als andere anzugeben; es hat aber alle walirscheinlichkeit, dass der dichter Florus - bei Charisius Amnius benamnt - aus der zeit 
des Hadrianus und unser verfasser [der epitome], wie auch allgemein angenommen wird, dieselbe person ist". Was übrigens hier Spengel von der „allgemein angenommenen" udentität beider sagt, bedarf der einsclıränkung. Unter den literarhistorikern spricht sich vielmehr Bernhardy (p. 707, anm. 5114) entschieden dagegen aus: es könne „nicht bezweifelt werden, dass der historiker verschieden vom dichter Florus, walirscheinlich aber von dem afrikaner P. Annius Florus nicht verschieden war". Teuffel (1) gedenkt einer einheit des historikers, des rhetors und des dichters mit keinem worte, und bei Hübner liesse sich auf eine identificirung der beiden ersteren nur daraus schliessen, weil er (Grundr. zu vorles. p. $64^{\mathbf{z}}$ ) in dem den historiker Julius Florus behandelnden \$. 98 kritische beitrage von Haupt zu dem rhetor erwähnt. Sauppe (nr. 22) hat die bezeichnung mit mehreren namen ganz vermieden. Rebers $(12$, p. 67) deutung, dass in dem titel des rhetorischen bruclistiicks P. Annii Flori das P. nicht praenomen sei, sondern poelu bedeute, ist zwar verwerflich; doch bemerkt er selbst im einklange mit Spengel richtig: „Mag man aus dem titel des fragmentes den namen des geschiclitschireibers in Publius verändern, oder Julius allein dafür setzen, oder annelımen, dass wir bezüglich des namens im unklaren sind, das darf man als fest behaupten, dass der fragmentist, der geschichtschreiler, der Florus des Spartianus und der des Charisius eine und dieselbe person war, wenn man auch zweifeln mag, wie sein eigentlicher name gelautet hat."

Auf die frage nach dem zeitalter des Florus ist bereits im vorausgehenden die antwort angedeutet; die identificirung des listorikers mit dem rhetor und dichter stützt sicb ja zunächst auf die annahme, dass derselbe in die zeit des Hadrianus gehörc. Diese annahme aber beruht auf deen zeugnisse der epitome selbst $(1,8)$ : a Caesare Augusto in secculum nostrum haut multo minus anni ducenti, quibus inertia Caesurum quesi consenuit adque decoxil, nisi quod sub Traieno principe movit lacertos et praeter spem omnium senechs imperii quasi reddila iuvenlute reviruit. Nachdem in diesen worten die lesart movit und reviruit durch $B$, mit welchem im ersten falle auch $N$ stimmt, gewährleistet ist, kann, wie selbst der vorsichtige Jahn olne vorbehalt ausspricht, kein zweifel darüber bestehen, dass Florus unter Hadrianus gelebt hat. Vossius (a. o.), welcher zwar jene schon längst vor auffindung des $B$ von manchen, besonders von J. Camers vorgezogene lesart kannte, alber sich für movet und revirescit erklärte, bezeichnete die letzten zeiten des Trajanus als die blütheperiode unseres scliriftstellers, der iibrigens iurh die folgende regierungszeit noch erlebt habe. Aber bedenken fasst Vossius, mit dessen annahme auch Ciossrau zusammentrifft, üler die chronologische berechnung bei Florus, die er zu berichtigen sucht, indem er statt CC vorschligt CL zu schreiben, was ziemlich genau dem zeitraume von 
Augustus' principat lis znun tode des Trajauns entspräclue. Dagegen wird festzulalten sein, was Heyn (nr. 13, p. 27) im allgemeinen über die chronologische ungenanigkeit des Florus lsemerkt : hand semel magnam harum revim inscientiam oo celare studet, quod numeris rotumdis ulilur. Auch Halm (ur. 2, p. IV) sugt bezüglich des prooemiums: numeros in codd. traditos, quamunam falsos asse rerum hisloria convincit, reslituendos pulavi, verilus ne correctionibus editorum admissis scriptorem ipsum corrigerem. Zum glücke ist der wortliut der oben ingefiilıten stelle des Florus so deutlich, dass die auslegung auch nicht durch die in der chronologischen angalue liegende unmöglichkeit verdunkelt werden kaun.

Der titel des geschichtswerkes ist in $B$ so iberliefert: EPITHOMA IVIA FIORI IOE TITO IIVIO - BELLOR VM OMINIII - ANNOR VII SEP'TINGENTOR VII IIIBRI - N - DVO FEI,ICITER, wälırend $N$ nur die kürzere bezeichunng EPITONı IDE TITO L.1VIO hat. Diss dieser "titel, der aus dem grunde angefochten worden ist, weil Florus sich keineswegs anf Livius als einzige quelle bescliränkt hat, „wenn nicht ursprïnglich, doch mindestens selir alt" sein müsse, erweist llommsen (Hermes I, 4(i8) durch das

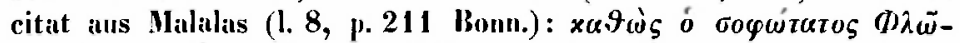

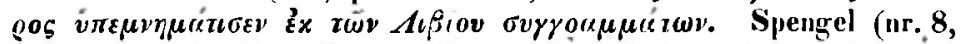
p. 32) hemerkt über diesen widerspruch zwischen titel und inhialt, indem er jenen offenhar für ursprunglich halt: ,vielleicht ist auch die hezeichunng des luches . . ilssichtlich gewahlt, um demselben melir cingang zu verschaffen, und bei aller allweichung konnte dieses um so eher stiltfinden, als mit deun gerülımten, ilıer den späteren viel zu ausgedehnten werke des Iivius gleicher umfung eingelialten wurde [uur dass bei Florus die vorgeschichte Roms felult]; desto hesser für den antor, wenn der vermeintliche auszug zugleich originelles darbot, und der leser melır fand als die bescheidenheit des titels ihn ahnen liess". Einfacher und treffender alber erklärt sich der auflallende aber nicht beispiellose ${ }^{2}$ ) titel der epilome aus dem von llommsen (vgl. a. o.) für die gesammte hehandlung der geschichte der repullik in der haiserzeit geführten naclıweis, "dass all diese späteren allrisse auszigge aus Livius entweder waren oder doch datür galten“.

Aus jener thatsache, dass Florus von Johannes Matalas sowolu lenutzt als auch cilirt worden ist, folgert Rühl (die verl,reitung des Justinus im mitlelalter p. 5), dass es eine griechische bearbeitung des Florus gegelen hale, da Malalis nur in einer solchen das luch kennen konnte. Dies wäre ein glianzendes zeugniss für die verbreitung des werkes, stimmt übrigens durchius zu

2) Man denke an die mit demselben recht oder unrecht sogenannten excerpta ex libris Scx. Aurelii Vicloris a Cacsare Aug. usique ad Theodosium imp. 
dem, was wir sonst hievon wissen. Zwar felılen nus zeugnisse darüber, welche aufnalıme die epitome bei den zeitgenossen gefunden laat; aber dass sie späterlin vielfachen ankling gehabt und formell als vorbild für die nachalımung, materiell als quelle für ausbeutnng im ganzen und einzelnen gedient hat, lässt sich erweisen. Ind dass dies nichit erst ,im vierten jalırlındert und später“s geschelıen ist, wie Spengel sagt (a. o. p. 32), sondern schon in der allernäclisten zeit und zwar wahırscheinlich unter Antoninus l'ius, wird in dem zweiten anliange an dem, was L. Ampelius aus Florus gesclïpft lat, gezeigt werden. Unter den späteren liat Festus (Sextus) RuIns in seinem Breviarium rerum gestarum populi Romani namentlich für die erzälılung orientalisclier kriege aus Florus geschöpft; als beispiel mag lier die stelle ïher den mordversucl gegen Caius Cuesar, den neffen des Augustus, angefülırt werden:

Flor. 11, 32 (44)

Fest. 19

quippe Dones, quem rex Artagerae Donnes quidam, quem Parthis Arex Parthis (artaxerses Parthis $B$, sacis praeposuerat, proditione siartax atis $N$ ) praefecerat, simulata mulata libellum, in quo conscripti proditione adortus virum intentum thesauri continerentur, optulit . libello, quem ut thensaurorum ra- qquem cum imperator Romanus letiones continentem ipse porrexerat. geret intentius, cultro adgressus stricto repente ferro subiit. Caium (codd. Gotl, et Baub. claudium) vulneravit.

Auch die oben mitgetheilte plirase des Florus (I, 8) hat Festus sich angeeignet (20): Traianus qui post Augustum Romanae rei publicae movit lacertos. Jener erste satz ist auch dirum charakteristisch, weil er zeigt, dass es selbst einem epitomator wie Festus leichter ist, die knappe darstellung des Florus zu paraphirasiren ials zu excerpiren.

Auffallende iibereinstimmung mit Florus zeigt im vierten jalirlundert iuch der iutor des schififtchens de viris illustribus urbis Romae, worauf Spengel (nr. 8, 32 f. anm. 2) und Heyn (nr. 13, p. 6 adnot.) aufmerksam machen, wälırend Jalın (nr. 1, p. XI,VIII) dirüber scliweigt. Spengel verzeichnet eine reilie von stellen, in welchen theilweise der wortlaut so ähnlich ist, dass z. b. Flor. I, 27 (2) Mommsens glänzende emendation ilıren beleg vir. ill. 55, 1 findet. Ferner stimmt der autor dieser schrift in einzelnen irrtliimern auf so bemerkenswertlie weise mit Florus überein, dass er nach der ansicht von Heyn (a. o.) nur diesem als seiner quelle gefolgt sein könnte. Aber es ist hier doch besondere vorsicht geboten. So berulit unter den von Spengel angefülirten analogien die ahnlichlıeit des wortlautes Flor. 1, 33, 15 und vir. ill. 71, 1 auf der gemeinsimen quelle Livius, wie ausser der a. o. citirten stelle aus Liv. epit. I. III anch Vell. Paterc. II, 1, 3 andeutet. Unter den von Ileyn (a. o.) beigebracliten parallelen, aus denen die 
ausbeutung des Florus in der schrift de vir. illustribus erhellen soll, ist z. b. Vir. ill. 2, 5 entschieden anders zu erklïren, da, angenommen dass der vulgattext echt ist, die hier genimnten Antemnates, Crustumini, Fidenates bei Flor. I, 1, $10 \mathrm{f}$, gar nicht vorkommen, also auch nicht aus ilum geschöpft sein können, da aber, falls die von Arntzen bentutzten handschriften zuverlassiger sind, gerade der von Heyn urgirte name Veientes dort zu streichen ist, sohin die stelle jede beweiskraft verliert. Vorsichtiger und richtiger als Heyn urtheilt daher Spengel, welcher (a. o.) zwar angibt, dass die der sonst daselbst eingehaltenen chronologischen ordnung widersprechende gruppirung von Menenius Agrippa, Marcius Coriolanus, Licinius Stolo und L. Virginius Vir. ill. 18-21 sich einfach aus der von Florus beliebten vereinigung dieser namen $I, 17$ unter dem titel de seditionibus erkläre; aber zugleich bemerkt, dass der autor der schrift de vir. ill. ,vollständigere quellen vor sich hatte" [oder die gleichen quellen vollständiger ausbeutete].

Dass Augustinus, obschon er Florus nirgends nennt, ihn dennoch gekannt und benutzt, gelegentlich auch (de civ. dei III, 19) kurz und treffend charakterisirt hat, ist wie von den früheren herausgebern des Florus so auch von Jahu (nr. 1, p. XLVIIII) und von Spengel (nr. 8, p. 18, anm. 2; vgl. p. 32) angedeutet worden. Elenso hat Orosius nicht nur den historischen inhalt sondern auch die rhetorische form der epitome stellenweise ausgebeutet. Insbesondere aber hat Jordanes sein werk de successione regnorum zum grossen theile bis zum zweiten macedonischen kriege so theu aus Florus ausgeschrieben, dass die besten handschriften seines werkes von Jahn und Halm mit grossem gewinue für die constituirung des textes der epitome verwerthet werden kounten. Dic ziemlich grosse zahl der erhaltencn hamlschriften des Florus so wie die mannichfache zerrüttung des textes weisen auch auf die fleissige lectüre des durch geringen umfang, ergreifende darstellung und edle moral sich empfehlenden büchleins während des mittelalters hin. Liutprand und Freculf sind nicht die einzigen schriftsteller dieser zeit, die Florus ausgebeutet haben.

Nlünnerstadt.

A. Eussner.

(Die fortsetzung folgt im nächsten hefte).

\section{Verg. Georg. IV, 330}

muss in fer slabulis inimicum ignem bei slabulis an die bienen (s. vs. 14. 191), und somit hei atque interfice messes ebenfalls an diese, also an honig (s. vs. 231) gedacht werden: daher steht vs. 331 sata für segeles, wie viles ausserdem verlangt. Auch pectrdum vs. 327 bezeichnet die bienen: s. vs. 168.

Ernst ron Leutscle. 